\title{
CONVERGENCE OF AN ALGORITHM FOR THE ANISOTROPIC AND CRYSTALLINE MEAN CURVATURE FLOW*
}

\author{
ANTONIN CHAMBOLLE ${ }^{\dagger}$ AND MATTEO NOVAGA
}

\begin{abstract}
We give a simple proof of convergence of the anisotropic variant of a well-known algorithm for mean curvature motion, introduced in 1992 by Merriman, Bence, and Osher. The algorithm consists in alternating the resolution of the (anisotropic) heat equation, with initial datum the characteristic function of the evolving set, and a thresholding at level $1 / 2$.
\end{abstract}

Key words. anisotropic mean curvature flow, heat equation, signed distance function

AMS subject classifications. 35K65, 35K55, 53C44

DOI. $10.1137 / 050629641$

1. Introduction: The algorithm. More than ten years ago, Merriman, Bence, and Osher [26] proposed the following algorithm for the computation of the motion by mean curvature of a surface. Given a closed set $E \subset \mathbb{R}^{N}$, they let $T_{h} E=\{u(\cdot, h) \geq$ $1 / 2\}$, where $u$ solves the following heat equation:

$$
\begin{cases}\frac{\partial u}{\partial t}(x, t)=\Delta u(x, t), & t>0, x \in \mathbb{R}^{N} \\ u(\cdot, 0)=\chi_{E} & (t=0) .\end{cases}
$$

Then, they let $E_{h}(t)=T_{h}^{[t / h]} E$ (with $[t / h]$ the integer part of $t / h$ ), and conjectured that $\partial E_{h}(t)$ converges to $\partial E(t)$, as $h \rightarrow 0$, where $\partial E(t)$ is the (generalized) evolution by mean curvature starting from $\partial E$.

The proof of convergence of this scheme was established by Evans [17] and Barles and Georgelin [2]. Other proofs were given by Ishii [22] and Cao [12], where the evolution in (1) was replaced by the convolution of $\chi_{E}$ with a more general symmetric kernel. This was generalized by Ishii, Pires, and Souganidis [23] to the case of the convolution with an arbitrary kernel (with some growth assumptions). This approach was also studied by Ruuth and Merriman [29] (see also [28]). Vivier [34] and Leoni [25] have considered other generalizations with (1) replaced with a time and space dependent anisotropic heat equation with a lower order term. The space dependence is an additional difficulty and it is not clear how what we will present could be adapted to such situations; on the other hand, in the two latter papers, "only" the case of Riemannian anisotropies is considered, in contrast to what we will study here.

We propose here to study the generalization of this algorithm to the so-called anisotropic and crystalline curvature motion, as defined in [21, 33, 32, 31]. We follow the definition in [10]: we consider $\left(\phi, \phi^{\circ}\right)$ a pair of mutually polar convex 1homogeneous functions in $\mathbb{R}^{N}$ (i.e., $\phi^{\circ}(\xi)=\sup _{\phi(\eta) \leq 1} \xi \cdot \eta, \phi(\eta)=\sup _{\phi^{\circ}(\xi) \leq 1} \xi \cdot \eta$; see [27]). These are assumed to be locally finite, and, to simplify, even. The pair

\footnotetext{
*Received by the editors April 20, 2005; accepted for publication (in revised form) December 12, 2005; published electronically March 3, 2006.

http://www.siam.org/journals/sima/37-6/62964.html

${ }^{\dagger}$ CMAP, CNRS UMR 7641, École Polytechnique, 91128, Palaiseau Cedex, France (antonin. chambolle@polytechnique.fr).

${ }^{\ddagger}$ Dipartimento di Matematica, Università di Pisa, via Buonarroti 2, 56127 Pisa, Italy (novaga@ dm.unipi.it).
} 
$\left(\phi, \phi^{\circ}\right)$ will be referred as the anisotropy. The local finiteness implies that there is a constant $c>1$ such that

$$
c^{-1}|\eta| \leq \phi(\eta) \leq c|\eta| \text { and } c^{-1}|\xi| \leq \phi^{\circ}(\xi) \leq c|\xi|
$$

for any $\eta$ and $\xi$ in $\mathbb{R}^{N}$. We refer to $[9,10]$ for the main properties of $\phi$ and $\phi^{\circ}$.

Being convex and 1-homogeneous, $\phi^{\circ}$ (and $\phi$ ) is also subadditive, so that the function $(x, y) \mapsto \phi(x-y)$ defines a distance - the " $\phi$-distance." For $E \subset \mathbb{R}^{N}$ and $x \in \mathbb{R}^{N}$, we denote by $\operatorname{dist}^{\phi}(x, E):=\inf _{y \in E} \phi(x-y)$ the $\phi$-distance of $x$ to the set $E$, and by

$$
d_{E}^{\phi}(x):=\operatorname{dist}^{\phi}(x, E)-\operatorname{dist}^{\phi}\left(x, \mathbb{R}^{N} \backslash E\right)
$$

the signed $\phi$-distance to $\partial E$, negative in the interior of $E$ and positive outside its closure. One easily checks that

$$
\left|d_{E}^{\phi}(x)-d_{E}^{\phi}(y)\right| \leq \phi(x-y) \leq c|x-y|
$$

for any $x, y \in \mathbb{R}^{N}$, so that $d_{E}^{\phi}$ is differentiable a.e. in $\mathbb{R}^{N}$. The former inequality shows moreover that $\nabla d_{E}^{\phi}(x) \cdot h \leq \phi(h)$ for any $h \in \mathbb{R}^{N}$ if $x$ is a point of differentiability; hence $\phi^{\circ}\left(\nabla d_{E}^{\phi}(x)\right) \leq 1$. If $\phi$ and $\phi^{\circ}$ are smooth, one shows quite easily that $d_{E}^{\phi}$ is differentiable at each point $x$ which has a unique $\phi$-projection $y \in \partial E$ (solving $\min _{y \in \partial E} \phi(x-y)$ ). In this case, $\nabla d_{E}^{\phi}(x)$ is given by $\nabla \phi\left((x-y) / d_{E}^{\phi}(x)\right.$ ), so that $\phi^{\circ}\left(\nabla d_{E}^{\phi}(x)\right)=1$. If $\phi, \phi^{\circ}$ are just Lipschitz-continuous, one still shows that $\phi^{\circ}\left(\nabla d_{E}^{\phi}(x)\right)=1$ a.e. in $\mathbb{R}^{N}$; see $[9,10]$ for details.

A Cahn-Hoffman vector field $n_{\phi}$ is a vector field on $\partial E$ such that $n_{\phi}(x) \in$ $\partial \phi^{\circ}\left(\nu_{E}(x)\right)=\partial \phi^{\circ}\left(\nabla d_{E}^{\phi}(x)\right)$ a.e. on $\partial E$, where $\partial \phi^{\circ}$ is the (0-homogeneous) subgradient of $\phi^{\circ}$ (see $\left.[27,16]\right)$ and $\nu_{E}$ is the (Euclidean) exterior normal to $\partial E$. If such a field is given in a neighborhood of $\partial E$, then it is characterized by

$$
\phi^{\circ}\left(n_{\phi}(x)\right)=1 \text { and } n_{\phi}(x) \cdot \nabla d_{E}^{\phi}(x)=1 \text { a.e. }
$$

This follows from Euler's identity, since $\phi^{\circ}$ is 1-homogeneous. In this case, $\kappa_{\phi}=\operatorname{div} n_{\phi}$ is a $\phi$-curvature of $\partial E$. The $\phi$-curvature flow is then an evolution $E(t)$ such that at each time, the velocity of $\partial E(t)$ is given by

$$
V=-\kappa_{\phi} n_{\phi}
$$

where $n_{\phi}$ is a Cahn-Hoffman vector field and $\kappa_{\phi}$ is the associated curvature. If $\phi, \phi^{\circ}$ are smooth (e.g., in $C^{2}(\Omega \backslash\{0\})$ ), then $n_{\phi}, \kappa_{\phi}$ are uniquely defined, whereas if $\phi, \phi^{\circ}$ are merely Lipschitz (when, for instance, the Wulff shape $\{\phi \leq 1\}$ is a convex polytope), then $n_{\phi}$ can be nonunique and the anisotropy is called crystalline $[33,9]$.

As easily shown by formal asymptotic expansion, the natural anisotropic generalization of the Merriman-Bence-Osher algorithm is as follows. Given $E$ a closed set with compact boundary in $\mathbb{R}^{N}$, we let $T_{h}(E)=\{x: u(x, h) \geq 1 / 2\}$, where $u(x, t)$ is the solution of

$$
\begin{cases}\frac{\partial u}{\partial t}(x, t) \in \operatorname{div}\left(\phi^{\circ}(\nabla u) \partial \phi^{\circ}(\nabla u)\right)(x, t), & t>0, x \in \mathbb{R}^{N} \\ u(\cdot, 0)=\chi_{E} & (t=0)\end{cases}
$$


The function $u(x, t)$ is well defined and unique by classical results on contraction semigroups [11]: if $E$ is compact, it corresponds to the flow in $L^{2}\left(\mathbb{R}^{N}\right)$ of the subdifferential of the functional $u \mapsto \int_{\mathbb{R}^{N}} \phi^{\circ}(\nabla u)^{2} / 2 d x$ if $u \in H^{1}\left(\mathbb{R}^{N}\right)$, and $+\infty$ otherwise. On the other hand, if $\mathbb{R}^{N} \backslash E$ is compact, one defines $u$ by simply letting $u=1+v$, where $v$ solves the same equation with initial data $\chi_{E}-1$.

We are interested in the limit of the discrete evolutions $t \mapsto E_{h}(t)=T_{h}^{[t / h]} E$, as $h \rightarrow 0$. Our main result is a result of consistency with suitable "regular" evolutions: it states that if there exists a regular evolution starting from $E$ in the sense of our Definition 2.1 (which is a variant of a definition first introduced in [9] and includes smooth evolutions when the anisotropy is smooth), then $E_{h}(t)$ converges to this evolution. This consistency result, together with the monotonicity of the scheme ( $E \subseteq F \Rightarrow T_{h} E \subseteq T_{h} F$, as follows from the comparison principle for (3)), yields convergence also to all generalized solutions defined (in the smooth case) using barriers $[7,8]$ or, equivalently, viscosity solutions $[14,15,4,5,3]$, as long as these are unique. Also, it yields the convergence of the scheme to crystalline evolutions, when the initial set is convex. Existence and uniqueness of such (regular and generalized) evolutions are established, in the convex case, in [6].

Another important consequence of our consistency result is a comparision principle for the regular evolutions of Definition 2.1, which follows from the monotonicity of the scheme. It gives an alternative proof of uniqueness for the convex crystalline evolutions studied in [6] (the original proof is based on [9]).

We observe that evolutions similar to (2) might also be obtained by convolution with appropriate kernels as studied by Ishii, Pires, and Souganidis [23]. However, a complete characterization of these motions in dimension higher than 2 is still not known (see $[29,30]$ in two dimensions).

Our evolution is also different from the evolutions considered by Leoni [25] (or Vivier [34]); in her paper, the heat equation (1) is replaced with an equation of the form $u_{t}=A(x, t): D^{2} u+H(x, t, D u)$. The resulting surface motion is a variant of the mean curvature motion, with an $(x, t)$ dependent velocity which is a function of a Riemannian curvature (depending on $A$ ) plus a lower order forcing term.

It would be interesting to prove a similar consistency result for the variational variant of (3), which is somehow simpler to solve numerically (in the truly nonlinear anisotropic cases): for $E \subset \mathbb{R}^{N}$ bounded, one would define $T_{h} E=\left\{u_{h} \geq 1 / 2\right\}$, where $u_{h}$ is the solution of (with $\Omega \ni E$ bounded or $\Omega=\mathbb{R}^{N}$ )

$$
\min _{u \in H^{1}(\Omega)} \int_{\Omega} \phi^{\circ}(\nabla u(x))^{2}+\frac{1}{h}\left(u(x)-\chi_{E}(x)\right)^{2} d x .
$$

Although it is likely that this variant produces the same evolution as the original scheme (it is true in the isotropic case, since $u_{h}$ is given by the convolution of $\chi_{E}$ with a radially symmetric kernel), we could not extend our proof in all cases to this new scheme.

Our proof follows the same idea as our recent proof of consistency [13] for (a generalization of) the variational algorithm of Almgren, Taylor, and Wang [1]. However, we have just learned that Goto, Ishii, and Ogawa $[20,24]$ have recently given a new proof of the convergence of the Merriman-Bence-Osher algorithm, in the isotropic case, which is very similar to the proof we give here.

2. The consistency result and some consequences. If $E \subset \mathbb{R}^{N}$ we say that $E$ satisfies the interior $r W_{\phi}$-condition if and only if for any $x \in \partial E$ there exist $y \in E$ 
with $\phi(x-y)=r$ and $\phi\left(x^{\prime}-y\right) \geq r$ for any $x^{\prime} \in \mathbb{R}^{N} \backslash E$. We say that $E$ satisfies the exterior $r W_{\phi}$-condition if $\mathbb{R}^{N} \backslash E$ satisfies the interior $r W_{\phi}$-condition.

We will show a consistency result with regular evolutions of (2), in the sense of the following definition.

Definition 2.1. We say that $t \mapsto E(t)$ is an $r W_{\phi}$-regular $\phi$-curvature flow on $\left[t_{0}, t_{1}\right], t_{0}<t_{1}$, if and only if

(i) for any $t \in\left[t_{0}, t_{1}\right], E(t)$ satisfies the interior and exterior $r W_{\phi}$-conditions;

(ii) there exists a bounded and relatively open neighborhood $A$ of $\bigcup_{t_{0} \leq t \leq t_{1}} \partial E(t) \times$ $\{t\}$ in $\mathbb{R}^{N} \times\left[t_{0}, t_{1}\right]$ such that $d(x, t):=d_{E(t)}^{\phi}(x)$ is Lipschitz in $A$;

(iii) there exists a vector field $n: A \rightarrow \mathbb{R}^{N}$ with $n \in \partial \phi^{\circ}(\nabla d)$ a.e. in $A$, and $\operatorname{div} n \in L^{\infty}(A)$; and

(iv) there exists $\bar{c}>0$ such that $|\partial d / \partial t-\operatorname{div} n| \leq \bar{c}|d|$ a.e. in $A$.

This definition, up to the additional requirement that $E(t)$ satisfies an interior and exterior $r W_{\phi}$-condition, is due to Bellettini and Novaga [9, Def. 2.2].

Such evolutions are known to exist if $\phi, \phi^{\circ}$, and $\partial E$ are smooth enough (for instance, in $\left.C^{3, \alpha}\left(\mathbb{R}^{N} \backslash\{0\}\right)[1]\right)$, or for any $\phi, \phi^{\circ}$, when the initial set $E$ is convex and satisfies an interior $r W_{\phi^{-}}$-condition (exterior is always true in the case of convex sets) [6]. They also exist in the purely crystalline case, i.e., when both $\phi$ and $\phi^{\circ}$ are piecewise linear in dimension $N=2[18,19,31]$ (see section 4 for an example).

Our main theorem states that the anisotropic Merriman-Bence-Osher scheme is consistent with such evolutions.

ThEOREM 2.2. Let $E$ be a regular flow in the sense of Definition 2.1, on a time interval $\left[t_{0}, t_{1}\right]$. Then, for any $t$ and $\tau$ with $t_{0} \leq t<t+\tau \leq t_{1}, \partial T_{h}^{[\tau / h]} E(t)$ converges to $\partial E(t+\tau)$ in the Hausdorff sense, as $h \rightarrow 0$.

The following corollary, also shown in [9], is obvious.

Corollary 2.3. Let $E, F$ be two flows in the sense of Definition 2.1, on $\left[t_{0}, t_{1}\right]$, and assume $E\left(t_{0}\right) \subseteq F\left(t_{0}\right)$. Then $E(t) \subseteq F(t)$ for all $t \in\left[t_{0}, t_{1}\right]$. In particular, if $E\left(t_{0}\right)=F\left(t_{0}\right)$, then $E(t)=F(t)$ for all $t \in\left[t_{0}, t_{1}\right]$.

The next corollary follows, with a standard proof (see $[4,5]$ ), from the monotonicity and consistency of the scheme.

Corollary 2.4. Assume $E \subset \mathbb{R}^{N}$ is a closed set with compact boundary such that the generalized $\phi$-curvature flow $E(t)$, starting from $E$, is uniquely defined on a time interval $[0, T)$ (e.g., $\phi, \phi^{\circ} \in C^{2}\left(\mathbb{R}^{N} \backslash\{0\}\right)$, and no fattening occurs [14]). Then $\partial T_{h}^{[t / h]} E(t) \rightarrow \partial E(t)$ in the Hausdorff sense for any $t<T$, as $h \rightarrow 0$. The same conclusion holds for any $\phi, \phi^{\circ}$ if $E$ is convex, by the uniqueness result in [6].

Let us observe that this result follows easily from Theorem 2.2 when evolutions according to Definition 2.1 are known to exist. If not (e.g., if $\phi, \phi^{\circ}$ are merely $C^{2}$ ), this is still true; however, the proof relies on a comparison with appropriate strict super- and subsolutions, defined according to obvious modifications of Definition 2.1 (as in [13]).

Remark 1 . In case $\phi, \phi^{\circ}$ are not even, Theorem 2.2 still holds, but (i) the signed distance to the interface $d_{E(t)}^{\phi}(x)$ must be defined, in Definition 2.1, in a nonsymmetric way, and (ii) the term $\partial \phi^{\circ}(\nabla u)$ in (3) must be replaced with $-\partial \phi^{\circ}(-\nabla u)$ (since $\nabla u$ has a reverse orientation with respect to the outer normal to the set $E$ ).

3. Proof of Theorem 2.2. The proof of Theorem 2.2 is divided into several steps. The idea is to build appropriate sub- and supersolutions to (3), by means of the function $d(x, t)$, and to compare $T_{h} E(t)$ with $E(t+h)$. 
These barriers will be built by means of the function $\gamma: \mathbb{R} \times[0,+\infty) \rightarrow[0,1]$, which solves the heat equation

$$
\begin{cases}\frac{\partial \gamma}{\partial \tau}(\xi, \tau)=\frac{\partial^{2} \gamma}{\partial \xi^{2}}(\xi, \tau), & \xi \in \mathbb{R}, \tau>0, \\ \gamma(\xi, 0)=Y(\xi), & \xi \in \mathbb{R}(\tau=0),\end{cases}
$$

where $Y=\chi_{[0,+\infty)}$ is the Heaviside function. It is well known that $\gamma$ is given by

$$
\gamma(\xi, \tau)=\frac{1}{2 \sqrt{\pi \tau}} \int_{-\infty}^{\xi} e^{-\frac{s^{2}}{4 \tau}} d s .
$$

In particular, one readily sees that it is self-similar: indeed, the change of variables $s^{\prime}=s / \sqrt{\tau}$ yields

$$
\gamma(\xi, \tau)=\frac{1}{2 \sqrt{\pi}} \int_{-\infty}^{\frac{\xi}{\sqrt{\tau}}} e^{-\frac{s^{\prime 2}}{4}} d s^{\prime}=\gamma\left(\frac{\xi}{\sqrt{\tau}}, 1\right)=: \gamma_{1}\left(\frac{\xi}{\sqrt{\tau}}\right) .
$$

We first show the following (obvious) result.

Lemma 3.1. For any $\varepsilon>0$, there exists $\tau_{0}>0$ such that if $0 \leq \tau \leq \tau_{0}$, then $\gamma(\varepsilon, \tau) \geq 1-\tau$.

Proof. We just need to observe that $\tau \mapsto \gamma(\varepsilon, \tau)$ is $C^{1}$ with derivative 0 at 0 . This derivative is indeed given by $\left(-\varepsilon / \tau^{3 / 2}\right) \gamma_{1}^{\prime}(\varepsilon / \sqrt{\tau})=\left(-\varepsilon / \tau^{3 / 2}\right) \exp \left(-\varepsilon^{2} /(4 \tau)\right)$. There exists $\tau_{0}$ such that it is in $[-1,0]$ for $\tau \leq \tau_{0}$; hence $\gamma(\varepsilon, \tau) \geq \gamma(\varepsilon, 0)-\tau$ if $\tau \in\left[0, \tau_{0}\right]$, which shows the lemma.

Let us now consider $E, r>0, t_{0} \leq t_{1}, A$, and the functions $d(x, t), n(x, t)$, as in Definition 2.1. Possibly reducing $r$, we can assume that $\{|d| \leq r\} \subset A$. Let us fix $t \in\left[t_{0}, t_{1}\right), \delta \in[0, r / 2]$ and let $F=\{d(\cdot, t) \leq \delta\}$. Let $u$ be the solution of

$$
\begin{cases}\frac{\partial u}{\partial \tau}(x, \tau) \in \operatorname{div}\left(\phi^{\circ}(\nabla u) \partial \phi^{\circ}(\nabla u)\right)(x, \tau), & \tau>0, x \in \mathbb{R}^{N}, \\ u(\cdot, 0)=\chi_{F}=Y(-d(\cdot, t)+\delta) & (\tau=0) .\end{cases}
$$

We first show the following result.

Lemma 3.2. For any $\varepsilon \in(0, r / 2)$, there exists $\tau_{0}>0$ (independent of $\delta$ ) such that $\tau \leq \tau_{0}$ yields $u(x, \tau) \leq \tau$ for any $x$ such that $d(x, t)-\delta=\varepsilon$.

Proof. Let us fix $x_{0} \in \mathbb{R}^{N} \backslash F$ with $d\left(x_{0}, t\right)-\delta=\varepsilon$. Since $E(t)$ satisfies the exterior $r W_{\phi^{-}}$-condition, the function $(d(\cdot, t)-\delta)$ is, outside $F$, equal to $\operatorname{dist}^{\phi}(\cdot, F)$. Hence, letting $W=\left\{x: \phi\left(x-x_{0}\right)<\varepsilon\right\}$, one sees that $W \cap F=\emptyset$. We deduce that $\chi_{F} \leq 1-\chi_{W}$ in $\mathbb{R}^{N}$, so that $u(\cdot, \tau) \leq 1-w(\cdot, \tau)$, where $w$ is the solution of

$$
\begin{cases}\frac{\partial w}{\partial \tau}(x, \tau) \in \operatorname{div}\left(\phi^{\circ}(\nabla w) \partial \phi^{\circ}(\nabla w)\right)(x, \tau), & \tau>0, x \in \mathbb{R}^{N}, \\ w(\cdot, 0)=\chi_{W} & (\tau=0) .\end{cases}
$$

This solution is explicitly given by $w(x, \tau)=U\left(\phi\left(x-x_{0}\right) / \varepsilon, \tau / \varepsilon^{2}\right)$, where $U(|x|, \tau)=$ $\tilde{U}(x, \tau)$ and $\tilde{U}$ is the (radial) solution of the heat equation $\partial \tilde{U} / \partial t=\Delta \tilde{U}$ with initial datum $\chi_{B_{1}}$, the characteristic function of the unit ball in $\mathbb{R}^{N}$. It is well known that

$$
\tilde{U}(x, \tau)=\frac{1}{\sqrt{4 \pi \tau}^{N}} \int_{\{|y| \leq 1\}} \exp \left(-\frac{|x-y|^{2}}{4 \tau}\right) d y
$$


so that

$$
U(|x|, \tau)=\frac{1}{\sqrt{4 \pi \tau}^{N}} \int_{\{|y| \leq 1\}} \exp \left(-\frac{\left(|x|-y_{1}\right)^{2}+\sum_{i=2}^{N} y_{i}^{2}}{4 \tau}\right) d y .
$$

Using arguments similar to the proof of the previous lemma (based on the fact that $U$ is smooth near $(\xi, \tau)=0,0$ and $\partial U / \partial t(0,0)=0)$, one sees that there exists $\tau_{0}>0$ such that if $\tau \leq \tau_{0}, U(0, \tau) \geq 1-\varepsilon^{2} \tau$; hence $w(0, \tau) \geq 1-\tau$ if $\tau \leq \tau_{0}^{\prime}=\varepsilon^{2} \tau_{0}$. We deduce that $u\left(x_{0}, \tau\right) \leq \tau$ if $\tau \leq \tau_{0}^{\prime}$, depending only on $\varepsilon$. This shows the lemma.

Let us fix $\varepsilon<r / 4$ and let us look for a supersolution of (6) on a time interval $[0, h], h$ small, of the form

$$
v(x, \tau)=\gamma(-d(x, t+\tau)+\delta+\bar{c} \bar{\varepsilon} \tau, \tau)+h,
$$

in $B=\bigcup_{0 \leq \tau \leq h}\{x: d(x, t)-\delta \leq \varepsilon, d(x, t+\tau)-\delta \geq-\varepsilon\} \times\{\tau\}$, where the constant $\bar{\varepsilon}$ will be made precise later on. We observe that since the speed of the motion is bounded at any time for $\tau$ small enough, if $h$ is small enough (depending only on $r, \varepsilon), B$ remains inside $\left\{(x, \tau) \in \mathbb{R}^{N} \times[0, h]: \delta-\varepsilon \leq d(x, t+\tau) \leq \delta+2 \varepsilon\right\}$, and $(0, t)+B \subset A$.

At $\tau=0, v(x, 0)=Y(-d(x, t)+\delta)+h$ is strictly larger than $\chi_{F}(x)=u(x, 0)$. If $0 \leq \tau \leq h$ and $d(x, t)-\delta=\varepsilon$, by Lemma 3.2 we have $u(x, \tau) \leq \tau \leq h \leq v(x, \tau)$, provided $h$ is small enough. If, on the other hand, $d(x, t+\tau)-\delta=-\varepsilon$, then by Lemma 3.1, still for $h$ small enough, $v(x, \tau)=\gamma(-d(x, t+\tau)+\delta+\bar{c} \bar{\varepsilon} \tau, \tau)+h \geq$ $\gamma(\varepsilon, \tau)+h \geq 1-\tau+h$; hence $v(x, \tau) \geq 1 \geq u(x, \tau)$. We find that $v \geq u$ on $\{(x, \tau) \in \partial B: \tau<h\}$, which is the parabolic boundary of $B$ (and, in fact, our proof even shows that $v \geq u$ in a neighborhood of this boundary).

Hence, to get that $v$ is a supersolution of (6) in $B$, one has to show that $\partial v / \partial \tau \geq$ $\operatorname{div} Z$ for some field $Z \in \phi^{\circ}(\nabla v) \partial \phi^{\circ}(\nabla v)$ inside $B$.

One has, a.e. in $B$,

$$
\begin{aligned}
\frac{\partial v}{\partial \tau}(x, \tau)=\left(-\frac{\partial d}{\partial t}(x, t+\tau)+\bar{c} \bar{\varepsilon}\right) \frac{\partial \gamma}{\partial \xi}(-d( & x, t+\tau)+\delta+\bar{c} \bar{\varepsilon} \tau, \tau) \\
+ & \frac{\partial \gamma}{\partial \tau}(-d(x, t+\tau)+\delta+\bar{c} \bar{\varepsilon} \tau, \tau)
\end{aligned}
$$

whereas

$$
\nabla v(x, \tau)=-\frac{\partial \gamma}{\partial \xi}(-d(x, t+\tau)+\delta+\bar{c} \bar{\varepsilon} \tau, \tau) \nabla d(x, t+\tau) .
$$

Using the assumption that $\phi^{\circ}$ is even, we see that $\phi^{\circ}(\nabla v)=\partial \gamma / \partial \xi\left(\right.$ since $\phi^{\circ}(\nabla d)=$ 1 a.e. in $\mathbb{R}^{N}$ ) and that $\partial \phi^{\circ}(\nabla v)=-\partial \phi^{\circ}(\nabla d)$ (since $\partial \gamma / \partial \xi>0$ and $\partial \phi^{\circ}$ is 0 homogeneous and odd). Let now

$$
Z(x, \tau)=-\frac{\partial \gamma}{\partial \xi}(-d(x, t+\tau)+\delta+\bar{c} \bar{\varepsilon} \tau, \tau) n(x, t+\tau)=\phi^{\circ}(\nabla v(x, \tau))(-n(x, t+\tau)) .
$$

Since (by assumption) $n(x, t+\tau) \in \partial \phi^{\circ}(\nabla d(x, t+\tau))=-\partial \phi^{\circ}(\nabla v(x, \tau))$ for a.e. $x$ in $\mathbb{R}^{N}$ and any $\tau \in(0, h)$, one has $Z(x, \tau) \in \phi^{\circ}(\nabla v) \partial \phi^{\circ}(\nabla v)(x, \tau)$. Since $\phi^{\circ}$ is 1homogeneous, Euler's identity yields $\nabla d \cdot n=\phi^{\circ}(\nabla d)=1$ as soon as $n \in \partial \phi^{\circ}(\nabla d)$. We deduce 
(8) $\operatorname{div} Z(x, \tau)=-\operatorname{div}\left[\frac{\partial \gamma}{\partial \xi}(-d(x, t+\tau)+\delta+\bar{c} \bar{\varepsilon} \tau, \tau) n(x, t+\tau)\right]$

$$
\begin{aligned}
=\frac{\partial^{2} \gamma}{\partial \xi^{2}} & (-d(x, t+\tau)+\delta+\bar{c} \bar{\varepsilon} \tau, \tau) \\
& -\frac{\partial \gamma}{\partial \xi}(-d(x, t+\tau)+\delta+\bar{c} \bar{\varepsilon} \tau, \tau)(\operatorname{div} n(x, t+\tau)) .
\end{aligned}
$$

Since we have $\partial d / \partial t \leq \operatorname{div} n+\bar{c}|d|$ in $B$, we deduce using (7) and (8) that

$$
\begin{aligned}
& \frac{\partial v}{\partial \tau}(x, \tau) \geq \operatorname{div} Z(x, \tau)-\frac{\partial^{2} \gamma}{\partial \xi^{2}}(-d(x, t+\tau)+\delta+\bar{c} \bar{\varepsilon} \tau, \tau) \\
& \quad+\bar{c}(\bar{\varepsilon}-|d(x, t+\tau)|) \frac{\partial \gamma}{\partial \xi}(-d(x, t+\tau)+\delta+\bar{c} \bar{\varepsilon} \tau, \tau)+\frac{\partial \gamma}{\partial \tau}(-d(x, t+\tau)+\delta+\bar{c} \bar{\varepsilon} \tau, \tau) .
\end{aligned}
$$

Now, $\gamma$ satisfies the heat equation, so that if $|d(x, t+\tau)| \leq \bar{\varepsilon}$ a.e. in $B$, we get

$$
\frac{\partial v}{\partial \tau}(x, \tau) \geq \operatorname{div} Z(x, \tau) .
$$

We choose $\bar{\varepsilon}=\delta+2 \varepsilon$ so that $|d(x, t+\tau)| \leq \bar{\varepsilon}$ a.e. in $B$ and (9) holds. By standard comparison results on parabolic equations (see [11]), we deduce that $v(x, h) \geq u(x, h)$. In particular, we have shown that there exists $h_{0}>0$ (depending only on $r, \varepsilon$ ) such that if $h<h_{0}$,

$$
\begin{aligned}
T_{h} F=\{u(\cdot, h) \geq & \left.\frac{1}{2}\right\} \subset\left\{v(\cdot, h) \geq \frac{1}{2}\right\} \\
& =\left\{x \in \mathbb{R}^{N}: d(x, t+h) \leq \delta+\bar{c} \bar{\varepsilon} h-[\gamma(\cdot, h)]^{-1}\left(\frac{1}{2}-h\right)\right\} .
\end{aligned}
$$

Since $\gamma_{1}(0)=1 / 2, \gamma_{1}^{\prime}(0)=1 /(2 \sqrt{\pi})$, we have $\gamma_{1}^{-1}(1 / 2-h)=-2 \sqrt{\pi} h+o(h)$. Now, $\gamma(\xi, h)=\gamma_{1}(\xi / \sqrt{h})$, so that $[\gamma(\cdot, h)]^{-1}=\sqrt{h} \gamma_{1}^{-1}$. We find that $[\gamma(\cdot, h)]^{-1}(1 / 2-h)=$ $(-2 \sqrt{\pi} h+o(h)) \sqrt{h}$. Hence, possibly reducing $h_{0}$, one gets that if $h<h_{0}$, then $[\gamma(\cdot, h)]^{-1}(1 / 2-h) \geq-4 h^{3 / 2}$. Recalling that $\bar{\varepsilon}=\delta+2 \varepsilon$, we find that if $h<h_{0}$,

$$
T_{h} F \subset\left\{x \in \mathbb{R}^{N}: d(x, t+h) \leq[\delta+(\bar{c}(\delta+2 \varepsilon)+4 \sqrt{h}) h]\right\} .
$$

Now, we deduce that $\left(\varepsilon \in(0, r / 4)\right.$ being fixed) if $t \in\left[t_{0}, t_{1}\right), h \leq h_{0}$, and $k \geq 1$ with $t+k h \leq t_{1}$, one has

$$
T_{h}^{k}(E(t)) \subset\left\{x \in \mathbb{R}^{N}: d(x, t+k h) \leq \delta_{k}\right\}
$$

with $\delta_{0}=0$ and $\delta_{k}=\delta_{k-1}+\left(\bar{c}\left(\delta_{k-1}+2 \varepsilon\right)+4 \sqrt{h}\right) h$, as long as $\delta_{k-1} \leq r / 2$. By induction, we find

$$
\delta_{k}=\left((1+\bar{c} h)^{k}-1\right)\left(2 \varepsilon+\frac{4 \sqrt{h}}{\bar{c}}\right) .
$$

In particular, if $\tau>0$ is fixed, with $t+\tau \leq t_{1}$, and $k=[\tau / h]$, we see that $\lim _{h \rightarrow 0} \delta_{k}=$ $2 \varepsilon\left(e^{\bar{c} \tau}-1\right)$. If $\varepsilon<r / 4$ is chosen small enough (less than $(r / 4) /\left(e^{\bar{c} \tau}-1\right)$ ), we see that for $h>0$ small enough, $\delta_{[\tau / h]} \leq r / 2$.

We now recall that any sequence of sets in $\mathbb{R}^{N}$ with equibounded boundaries has a subsequence that converges in the Hausdorff sense to a closed set. If $E^{\prime}$ is the Hausdorff limit of a converging subsequence of $T_{h}^{[\tau / h]} E(t)$, as $h \rightarrow 0$, we deduce that $E^{\prime} \subseteq\left\{d(\cdot, t+\tau) \leq 2 \varepsilon\left(e^{\bar{c} \tau}-1\right)\right\}$. Since this must be true for all $\varepsilon>0$ small enough, 
one sees that $E^{\prime} \subseteq E(t+\tau)$. On the other hand, a symmetric argument (based on subsolutions of the equation) will yield that if $\mathbb{R}^{N} \backslash E^{\prime \prime}$ is the Hausdorff limit of a converging subsequence of $\left(\mathbb{R}^{N} \backslash T_{h}^{[\tau / h]} E(t)\right)_{h>0}$, then $\mathbb{R}^{N} \backslash E^{\prime \prime} \subseteq \overline{\mathbb{R}^{N} \backslash E(t+\tau)}$; that is, $\operatorname{int}(E(t+\tau)) \subseteq E^{\prime \prime}$. Without loss of generality, one can choose the same subsequence in both limits above: in this case, one can show that $E^{\prime \prime} \subset E^{\prime}$, and $E^{\prime} \backslash E^{\prime \prime}$ is the Hausdorff limit of $\partial T_{h}^{[\tau / h]} E(t)$ (which might differ from $\partial E^{\prime}$ or $\partial E^{\prime \prime}$ ). Since $\operatorname{int}(E(t+\tau)) \subseteq E^{\prime \prime} \subset E^{\prime} \subseteq E(t+\tau)$, we see that $E^{\prime \prime}=\operatorname{int}(E(t+\tau)), E^{\prime}=$ $E(t+\tau), E^{\prime} \backslash E^{\prime \prime}=\partial E(t+\tau)$, and by uniqueness of this Hausdorff limit we deduce Theorem 2.2.

4. A numerical example. The algorithm is quite easy to implement numerically. Of course, there is some difficulty in computing precisely the solution of (3) in strongly anisotropic or crystalline cases, especially when the subgradient $\partial \phi^{\circ}$ is multivalued. We experimented with an implicit method, based on iterative resolutions of the variational problem (4). More precisely, we approximate $u(\cdot, h)$ with $w_{n}(x)$, where $h=n h^{\prime}, n$ is a fixed (small) integer, $w_{0}=\chi_{E}$, and for $i=0, \ldots, n-1, w_{i+1}$ solves (in a domain $\Omega$ "large" with respect to $E$ )

$$
\min _{w \in H^{1}(\Omega)} \int_{\Omega} \phi^{\circ}(\nabla w(x))^{2}+\frac{1}{h^{\prime}}\left(w(x)-w_{i}(x)\right)^{2} d x .
$$

To solve this minimization problem in the crystalline case, we discretize (here, on a bidimensional finite differences grid) and solve the dual problem (see, for instance, [16])

$$
\min _{\xi \in L^{2}\left(\Omega ; \mathbb{R}^{N}\right)} \int_{\Omega} \phi(\xi(x))^{2}+h^{\prime}\left(\left(w_{i}(x) / h^{\prime}\right)-\operatorname{div} \xi(x)\right)^{2} d x,
$$

using a conjugate-gradient method. Then, $w_{i+1}=w_{i}-h^{\prime} \operatorname{div} \xi$. The thresholding at level $1 / 2$ is replaced by a "soft thresholding" $w_{n}(x) \mapsto \min \left\{1, \max \left\{1 / 2+\sigma\left(w_{n}(x)-\right.\right.\right.$ $1 / 2), 0\}\}$, where $\sigma$ is adapted to the spatial discretization step, in order to keep a precision slightly higher than the grid's. In the example shown in Figure 1, the Wulff shape $\{\phi \leq 1\}$ is a hexagon.

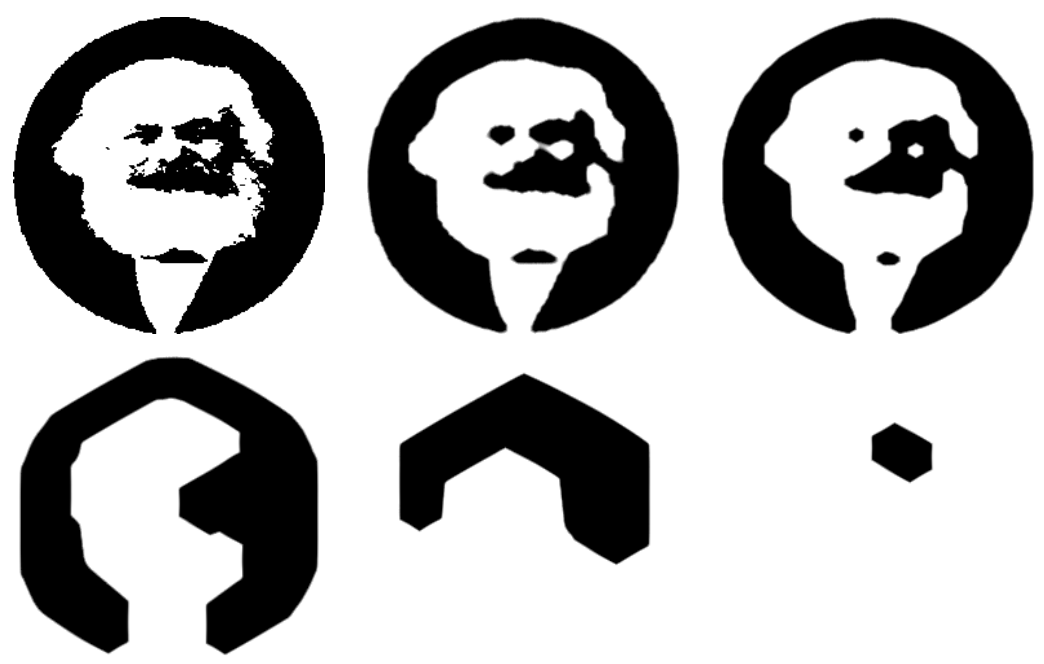

FIG. 1. Evolutions at times $t=0,5,25,60,400,800$. 


\section{REFERENCES}

[1] F. Almgren, J. E. Taylor, And L.-H. Wang, Curvature-driven flows: A variational approach, SIAM J. Control Optim., 31 (1993), pp. 387-438.

[2] G. Barles and C. Georgelin, A simple proof of convergence for an approximation scheme for computing motions by mean curvature, SIAM J. Numer. Anal., 32 (1995), pp. 484-500.

[3] G. Barles, H. M. Soner, And P. E. Souganidis, Front propagation and phase field theory, SIAM J. Control Optim., 31 (1993), pp. 439-469.

[4] G. BARles And P. E. Souganidis, Convergence of approximation schemes for fully nonlinear second order equations, Asymptot. Anal., 4 (1991), pp. 271-283.

[5] G. Barles And P. E. Souganidis, A new approach to front propagation problems: Theory and applications, Arch. Ration. Mech. Anal., 141 (1998), pp. 237-296.

[6] G. Bellettini, V. Caselles, A. Chambolle, and M. Novaga, Crystalline mean curvature flow of convex sets, Arch. Ration. Mech. Anal., 179 (2006), pp. 109-152.

[7] G. Bellettini and M. Novaga, Minimal barriers for geometric evolutions, J. Differential Equations, 139 (1997), pp. 76-103.

[8] G. Bellettini and M. Novaga, Comparison results between minimal barriers and viscosity solutions for geometric evolutions, Ann. Scuola Norm. Sup. Pisa Cl. Sci. (4), 26 (1998), pp. $97-131$.

[9] G. Bellettini And M. Novaga, Approximation and comparison for nonsmooth anisotropic motion by mean curvature in $\mathbf{R}^{N}$, Math. Models Methods Appl. Sci., 10 (2000), pp. 1-10.

[10] G. Bellettini And M. Paolini, Anisotropic motion by mean curvature in the context of Finsler geometry, Hokkaido Math. J., 25 (1996), pp. 537-566.

[11] H. BRÉzIS, Opérateurs maximaux monotones et semi-groupes de contractions dans les espaces de Hilbert, North-Holland Math. Stud. 5, Notas de Matemática (50), North-Holland, Amsterdam, 1973.

[12] F. CAO, Partial differential equations and mathematical morphology, J. Math. Pures Appl. (9), 77 (1998), pp. 909-941.

[13] A. Chambolle and M. Novaga, Implicit Time Discretization of the Mean Curvature Flow with a Discontinuous Forcing Term, preprint CMAP 571, École Polytechnique, France, 2005.

[14] Y. G. Chen, Y. Giga, And S. Goto, Uniqueness and existence of viscosity solutions of generalized mean curvature flow equations, Proc. Japan Acad. Ser. A Math. Sci., 65 (1989), pp. 207-210.

[15] M. G. Crandall, H. Ishit, and P.-L. Lions, User's guide to viscosity solutions of second order partial differential equations, Bull. Amer. Math. Soc. (N.S.), 27 (1992), pp. 1-67.

[16] I. Ekeland And R. Témam, Convex Analysis and Variational Problems, Classics Appl. Math. 28, SIAM, Philadelphia, 1999.

[17] L. C. Evans, Convergence of an algorithm for mean curvature motion, Indiana Univ. Math. J., 42 (1993), pp. 533-557.

[18] M.-H. Giga And Y. Giga, Generalized motion by nonlocal curvature in the plane, Arch. Ration. Mech. Anal., 159 (2001), pp. 295-333.

[19] Y. Giga And M. E. GuRTin, A comparison theorem for crystalline evolution in the plane, Quart. Appl. Math., 54 (1996), pp. 727-737.

[20] Y. Goto, K. IshiI, And T. OGawa, Method of the distance function to the Bence-MerrimanOsher algorithm for motion by mean curvature, Commun. Pure Appl. Anal., 4 (2005), pp. 311-339.

[21] M. E. GuRTin, Thermomechanics of Evolving Phase Boundaries in the Plane, Oxford Mathematical Monographs, The Clarendon Press, Oxford University Press, New York, 1993.

[22] H. IsHiI, A generalization of the Bence, Merriman and Osher algorithm for motion by mean curvature, in Curvature Flows and Related Topics (Levico, 1994), GAKUTO Internat. Ser. Math. Sci. Appl. 5, Gakkōtosho, Tokyo, 1995, pp. 111-127.

[23] H. IshiI, G. E. Pires, AND P. E. Souganidis, Threshold dynamics type approximation schemes for propagating fronts, J. Math. Soc. Japan, 51 (1999), pp. 267-308.

[24] K. IshiI, Optimal Rate of Convergence of the Bence-Merriman-Osher Algorithm for Motion by Mean Curvature, preprint 16, available online at http://cpde.iac.rm.cnr.it (2004).

[25] F. LEONI, Convergence of an approximation scheme for curvature-dependent motions of sets, SIAM J. Numer. Anal., 39 (2001), pp. 1115-1131.

[26] B. Merriman, J. K. Bence, And S. J. Osher, Diffusion generated motion by mean curvature, in Computational Crystal Growers Workshop, J. E. Taylor, ed., Sel. Lectures Math., AMS, Providence, RI, 1992, pp. 73-83. 
[27] R. T. Rockafellar, Convex Analysis. Reprint of the 1970 Original, Princeton Landmarks in Mathematics, Princeton University Press, Princeton, NJ, 1997.

[28] S. J. RuUth, Efficient algorithms for diffusion-generated motion by mean curvature, J. Comput. Phys., 144 (1998), pp. 603-625.

[29] S. J. Ruuth and B. Merriman, Convolution-generated motion and generalized Huygens' principles for interface motion, SIAM J. Appl. Math., 60 (2000), pp. 868-890.

[30] S. J. RuUth and B. MerRiman, Convolution-thresholding methods for interface motion, J. Comput. Phys., 169 (2001), pp. 678-707.

[31] J. E. TAYLOR, Motion of curves by crystalline curvature, including triple junctions and boundary points, in Differential Geometry: Partial Differential Equations on Manifolds (Los Angeles, CA, 1990), Proc. Sympos. Pure Math. 54, AMS, Providence, RI, 1993, pp. 417438.

[32] J. E. TAYLOR, Surface motion due to crystalline surface energy gradient flows, in Elliptic and Parabolic Methods in Geometry (Minneapolis, MN, 1994), A. K. Peters, Wellesley, MA, 1996, pp. 145-162.

[33] J. E. Taylor, J. W. Cahn, and C. A. Handwerker, Geometric models of crystal growth, Acta Metall., 40 (1992), pp. 1443-1474.

[34] L. Vivier, Convergence of an approximation scheme for computing motions with curvature dependent velocities, Differential Integral Equations, 13 (2000), pp. 1263-1288. 